\title{
Anticancer effect of nucleoline-aptamer-conjugated gemcitabine loaded atellocollagen (10401) in pancreatic cancer patient-derived orthotropic xenograft model
}

\author{
$\underline{\text { Ji Su KIM }}{ }^{1,2}$, Sang Suk KIM${ }^{3}$, Ha Young WOO ${ }^{4}$, Joong Hwan LEE ${ }^{5}$, Chang Moo KANG*1,2 \\ 'Department of Hepatobiliary and Pancreatic Surgery, Yonsei University College of Medicine, Seoul, Korea \\ ${ }^{2}$ Pancreatobiliary Cancer Center, Yonsei Cancer Center, Severance Hospital, Seoul, Korea \\ ${ }^{3}$ Department of Animal Testing, CNBiologics Company, Yongin, Korea \\ ${ }^{4}$ Department of Pathology, Yonsei Cancer Center, Severance Hospital, Seoul, Korea \\ ${ }^{5}$ Department of Development, Interoligo Corporation, Anyang, Korea
}

Introduction: We investigated the anticancer effect and systemic effect of the atelocollagen (AC) patch coated nucleoline-aptamer-conjugated Gemcitabine (IO401 patch) by directly implanting to the tumor cell in pancreatic cancer patient-derived xenograft (PDX) model to purpose a future potential adjuvant surgical strategy during curative pancreatic resection for pancreatic cancer.

Methods: Pancreatic cancer PDX model was established. Animals were grouped randomly (7 mice per group) into three types of patch transplantation groups: G1 = Null AC patch, G2 = Gemcitabine AC patch, G3 = IO401 patch. Tumor volume (length $\times$ width ${ }^{2}$, $\mathrm{mm}^{3}$ ), Tumor weight $(\mathrm{mg})$, and Tumor inhibition rate $[1-(\mathrm{Ti}-\mathrm{To}) /($ average tumor volume of group $) \times 100$, Ti $=$ endpoint tumor volume, To = start tumor volume] were calculated. Anticancer therapy-related toxicity was evaluated by hematologic and histological findings.

Results: G3 (IO401 patch) showed the most significant reduction of tumor growth and tumor weight comparing with G1 (Null AC patch) and G2 (Gemcitabine AC patch) $(p=0.014, p=0.018)$. G3 also showed the most significant tumor inhibition rate comparing with G1 and G2 $(p=0.011)$. G2 and G3 has the low necrosis proportion in histological finding comparing with G1 $(p=0.005, p<0.05)$. Moreover, no leukopenia, no anemia, and no neutropenia were observed in G3.

Conclusions: We demonstrated the anticancer effect of the IO401 patch by directly implanting to tumor cell in pancreatic cancer PDX model. This directaly implantable aptaber-drug conjugate system on tumor cell is expected to be a new surgical strategy to further increase the oncological importance of margin negative resection in pancreatic cancer surgery. Further research will be needed. 